Artigo Original

\title{
Validação para língua portuguesa: Lista de Checagem da Movement Assessment Battery for Children
}

\author{
Maria Helena da Silva Ramalho ${ }^{1}$ \\ Nadia Cristina Valentini ${ }^{2}$ \\ Caren Fernanda Muraro ${ }^{3}$ \\ Ramona Gadens ${ }^{4}$ \\ Glauber Carvalho Nobre ${ }^{4}$ \\ ${ }^{1}$ Centro de Ciências da Saúde e do Esporte, Universidade do Estado de Santa Catarina, \\ Florianópolis, SC, Brasil \\ ${ }^{2}$ Escola de Educação Física, Universidade Federal do Rio Grande do Sul, \\ Porto Alegre, Brasil \\ ${ }^{3}$ Programa de Pós-graduação Stricto Sensu, Ciências do Desporto, Universidade de \\ Trás-Os-Montes e Alto Douro, Vila Real, Portugal \\ ${ }^{4}$ Laboratório de Instrumentação, Centro de Ciências da Saúde e do Esporte, Universidade \\ do Estado de Santa Catarina, Florianópolis, SC, Brasil
}

Resumo: A Lista de Checagem do Movement Assessment Battery for Children segunda edição (LC-MABC2) foi desenvolvida como instrumento de triagem para crianças com dificuldades de movimento, mais especificamente com DCD. Objetivo: Traduzir, adaptar e verificar a validade de face, conteúdo e construto e a fidedignidade da versão em Português da LC-MABC-2; e, verificar a utilidade do referido instrumento de triagem no Brasil. Metodologia: Participaram 47 profissionais da saúde (educadores físicos e fisioterapeutas) e 20 pais; e, 532 crianças, (meninas: 276; meninos: 256) entre 5 e 12 anos. Resultados: indicam que a versão portuguesa adaptada da LC-MABC-2 demonstrou valores de concordância elevados para clareza e pertinência; validade convergente e descriminante apropriada; e, índices de confiabilidade (escore total, $\alpha=0,94$ ) e objetividade inter-avaliadores elevada. Conclusões: A versão em português do LC-MABC-2 demonstrou ser válida e fidedigna na triagem de crianças com dificuldades motoras para encaminhamento para avaliações mais detalhadas e possível intervenção.

Palavras-chave: Estudos de validação. Destreza motora. Desenvolvimento infantil.

\section{Validation for portuguese language: Movement Assessment Battery for Children' Checklist}

\begin{abstract}
The checklist of the Movement Assessment Battery for Children second edition (MABC-2Checklist) was developed as an instrument for screening children with motor difficulties. Objective: To translate, adapt and verify (1) the face, content and construct validity and the reliability of the Portuguese version of the MABC-2 Checklist; as well as to investigate the usefulness of the MABC-2-Checklist as a screening instrument in Brazil. Methods: Participated in the study 47 health related professional (physical educators and physical therapists), 20 parents, and 532 children (girls: 276; boys: 256) between 5 and 12 year-old. Results: indicated that the adapted version of the MABC-2-Checklist to Portuguese demonstrated convergent and discriminant validity ( $p$ values $\leq 0,05$ ); excellent reliability (all sections, $\alpha=0,94$ ) and high indices of inter-evaluators reliability. Conclusion: the adapted Portuguese version of the Checklist-MABC-2 demonstrated validity and reliability to screen children with motor difficulties and to further refereed to more complete assessments and intervention.
\end{abstract}

Keywords: Validation study. Motor skills. Childhood development.

\section{Introdução}

$\begin{array}{ccc}\text { Crianças com } & \text { Desordem } & \text { Coordenativa } \\ \text { Desenvolvimental } & \text { (DCD) } & \text { apresentam }\end{array}$
desempenhos motores abaixo do esperado para sua idade de desenvolvimento, o qual não é decorrente de nenhum problema de saúde (GEUZE, 2005; SUMMERS et al., 2008). O atraso no desenvolvimento de marcos motores interfere nas atividades físicas diárias ( $\underline{\text { SCHOTT }}, 2007)$, e estas crianças tendem a evitar a prática de esportes (CAIRNEY et al., 2010; MISSIUNA et al., 2007). As consequências possíveis em curto prazo podem estender-se através dos anos aumentando os riscos de comorbidades associada à desordem como obesidade (MISSIUNA et al., 2007), depressão e isolamento 
social (CUMMINS et al., 2005; POULSEN et al., 2007) e, desempenhos escolares inferior aos seus pares (AMERICAN PSYCHIATRIC ASSOCIATION, 2000; GIBBS et al., 2007).

A detecção e intervenção precoce para crianças com DCD é essencial uma vez que esta desordem pode repercutir em dificuldades motoras na vida adulta (COUSIN; SMYTH, 2003; VISSER, 2003). Crianças vulneráveis podem resistir aos efeitos negativos de fatores de risco (MAHONEY; et al., 2004) se estratégias interventivas adequadas são propiciadas (HENDERSON et al., 2007; SCHOEMAKER et al. 2003; VALENTINI et al., 2008). Especificamente profissionais da saúde possuem recursos instrumentais que permitem a identificação desta desordem, entretanto estes profissionais, em geral, só terão acesso às crianças que foram recomendadas para tratamento. Em contrapartida, professores convivem com a oportunidade de observar uma grande variedade de habilidades motoras nas salas de aula, no pátio durante brincadeiras e nas aulas de educação física. A identificação sistemática destas crianças depende também do acesso de professores e terapeutas aos instrumentos de triagem adequados e validados para uma especifica cultura (DIDO, 2005; VALENTINI et al., 2008). O uso de instrumentos de triagem propiciam informações relativamente rápidas sobre a competência motora de crianças, embora seja menos objetivo quando comparado com os testes normatizados (HENDERSON et al., 2007; DIDO, 2005; SCHOEMAKER et al., 2003).

Até o presente momento alguns questionários têm sido utilizados para a identificação de crianças com dificuldades motoras: o Motor Observation Questionnaire for Teachers (MOQ-T) (SCHOEMAKER, et al., 2003), o Developmental Coordination Disorder Questionnaire (DCDQ) desenvolvido no Canadá (PRADO et al., 2009) e a LC-MABC-2 (HENDERSON et al., 2007). Destaca-se que o DCDQ foi recentemente validado no Brasil (PRADO et al., 2009), enquanto que não há estimativas das propriedades psicrométricas LC-MABC-2 para crianças brasileiras, embora o mesmo esteja em corrente uso clinico e em pesquisas (FERREIRA et al., 2008a; 2008b; SILVA et al., 2006). Não obstante ao potencial da LC-MABC-2 como instrumento de triagem de crianças com provável DCD se faz necessário a verificação de sua validade no Brasil. Instrumentos podem sofrer interferência em seus resultados frente à adaptação à outro meio e à fatores socioeconômicos, étnicos e culturais diferentes (CRONBACH, 1996; FACHEL; CAMEY 2003; PASQUALI, 2001; VALLERAND, 1989).

Informações sobre as propriedades psicométricas da LC-MABC-2 são reportadas no manual (HENDERSON et al. 2007). Os autores reportam resultados satisfatórios para o uso do escore motor total e categorização dos escores com pontes de corte para normalidade, risco e dificuldades motoras. Validade discriminante para o LC-MABC-2 foi reportada em dois estudos, um com crianças com DCD (BARNETT et al. et al., 2007) e outro com crianças com síndrome de Asparger (SIAPERAS et al., 2007); os dois estudos propiciam evidencias de validade de critério para os pontos de corte da LC-MABC-2. Entretanto análises de consistência interna para a LC-MABC-2 não são reportadas. Portanto, o objetivo do presente estudo foi traduzir, adaptar e verificar a validade de face, conteúdo e construto e a fidedignidade da versão em Português da LCMABC-2; e, verificar a utilidade da LC-MABC-2 como instrumento de triagem no Brasil para diferentes idades.

\section{Método}

\section{Participantes}

Estudo aprovado pelo Comitê de Ética em Pesquisa ( $n^{\circ}$ 2008018). Participaram 4 tradutores, 3 doutores em movimento humano (painel de especialistas), 20 pais, 20 professores de sala de aula, 10 professores de educação física e 10 fisioterapeutas. $O$ número de tradutores, especialistas e público alvo (pais, professores de sala de aula, educadores físicos e fisioterapeutas) seguem as recomendações para validação transcultural de instrumentos (BEATON et al., 2000; HERNANDEZ-NIETO, 2002; VALLERAND, 1989). Foram analisadas 532 crianças, sendo 276 meninas, e 256 meninos, entre 5 e 12 anos ( $M=$ 8,75 , DP $=2,04$ ) foram incluídas de forma consecutiva, mediante o consentimento dos pais e ou pelos responsáveis legais (Tabela 1). 0 tamanho da amostra foi determinado com base nas recomendações de literatura em psicometria com no mínimo 10 participantes por item do instrumento (DASSA, 1999). Todos os participantes provêm da região sul do país (Santa Catarina e Rio Grande do Sul). 
Tabela 1. Numero de Participantes por faixa etária e sexo

\begin{tabular}{lccc}
\hline & \multicolumn{2}{c}{ SEXO } \\
\hline Idade (anos) & $\mathbf{N}(\%)$ & Meninas & Meninos \\
\hline 5 & $28(5.3)$ & $15(5.4)$ & $13(5.1)$ \\
6 & $60(11.3)$ & $26(9.4)$ & $34(13.3)$ \\
7 & $76(14.3)$ & $36(13)$ & $40(15.6)$ \\
8 & $76(14.3)$ & $41(14.9)$ & $35(13.7)$ \\
9 & $93(17.5)$ & $50(18.1)$ & $43(16.8)$ \\
10 & $68(12.8)$ & $31(11.2)$ & $37(14.5)$ \\
11 & $74(13.9)$ & $43(15.6)$ & $31(12.1)$ \\
12 & $57(10.7)$ & $34(12.3)$ & $23(9)$ \\
\hline Total & $532(100)$ & $276(100)$ & $256(100)$ \\
\hline
\end{tabular}

\section{Instrumentos}

A LC-MABC-2 (HENDERSON et al., 2007) é um instrumento de triagem, administrado por adultos, para identificar crianças (entre 5 e 12 anos) com dificuldades motoras. A LC-MABC-2 é composta de 3 seções, listando comportamentos motores observados no cotidiano da criança, em casa e na escola (HENDERSON et al., 2007). A observação dos comportamentos leva aproximadamente 10 minutos quando conduzidas por adultos familiarizadas com a criança. $\mathrm{Na}$ seção A do instrumento observam-se comportamentos motores com a criança parada em um ambiente previsível; e, na seção B comportamentos motores da criança em movimento e em um ambiente dinâmico. Os escores nas seções A e B são organizados em uma escala Likert com valores de 0, 1, 2 e 3 referentes a qualidade da execução do movimento. Primeiramente decide-se se a criança executa a tarefa; e se o fez muito bem (escore 0) ou somente bem (escore 1). Se a criança não é capaz de realizar o movimento deve ser observado se a mesma esta próxima a realizar (escores 2) ou nem perto de realizar (escore 3) as tarefas. $O$ instrumento propicia um Escore Motor Total (EMT) composto pela soma da seção A e B; categorização do comportamento motor em: Competência Motora (CM) apropriada para a idade; Risco de Atrasos Motores (RAM) quando demonstra alguns problemas de movimento menores que precisam ser monitorados; e, com Dificuldades Motoras Graves (DMG). A seção C contém itens sobre fatores que possivelmente afetam negativamente 0 movimento, estas questões são organizadas em repostas dicotômicas (HENDERSON et al., 2007).

Uma Escala do tipo Likert com 5 pontos foi utilizada na avaliação da Clareza e Pertinência aos itens da LC-MABC-2 pelo painel de especialistas e publico alvo; partindo de "pouquíssima pertinência/clareza", resposta 1 , até "muitíssima pertinência/clareza", resposta 5.

\section{Procedimentos}

Tradutores qualificados realizaram a tradução da LC-MABC-2. Primeiramente foram realizadas duas traduções (2 tradutores bilíngues independentes) do inglês para o português, resultando em duas versões independentes em português. Utilizando-se das duas primeiras traduções, o instrumento foi reconvertido ao idioma de origem por 2 outros profissionais bilíngues, resultando em 2 novas versões em inglês do teste. Em comitê os 4 tradutores e um dos pesquisadores elaboraram a versão final do questionário utilizando as versões individuais (VALLERAND, 1989; BEATON, et al., 2000; HERNANDEZ-NIETO, 2002).

A validade de conteúdo foi determinada por um painel de especialistas, que participaram da avaliação dos critérios motores da LC-MABC-2. Os especialistas receberam a versão final do questionário em português com a escala Likert para cada questão; e, pontuaram individualmente as questões quanto à clareza e pertinência (VALLERAND, 1989).

A validade de face foi realizada pelo públicoalvo para o uso do instrumento. Individualmente, pais, professores, educadores físicos, e fisioterapeutas expressaram suas opiniões quanto a clareza, praticidade, adequação, e linguagem dos itens (PASQUALI, 2001; VALLERAND, 1989). A aplicação da LC-MABC-2 (HENDERSON et al., 2007), foi realizada nas escolas por 3 professores de Educação Física, com experiência prática de ensino de crianças e adolescentes (entre 5 e 10 anos de atuação na área). Os professores estavam familiarizados com as crianças, pois atuavam nas escolas observando suas rotinas $e$ anotando suas dificuldades e ou conquistas motoras por aproximadamente um ano. Para completar a lista de checagem o tempo de observação de cada criança foi de aproximadamente 10 minutos.

\section{Analise dos dados}

Para a Validade de Conteúdo foram utilizados o Índice de Validade de Conteúdo (IVC) e teste de Wilcoxon (comparação de variáveis quantitativas entre dois grupos dependentes) e Friedman como post hoc teste para o teste de Wilcoxon. Para a análise de Validade de Face foi utilizado 
percentagem de concordância entre avaliadores. $\mathrm{Na}$ Validade do Construto foram utilizadas para a validade convergente correlações no EMT; e, para validade discriminante 0 teste de Wilcoxon (comparações seções A e B), o teste de Kruskal Wallis (comparações na categorização e nos grupos de idade no EMT) com testes de post hoc de Mann-Whitney U e correção de Bonferroni; e, $\mathrm{Eta}^{2}$ para a associação entre categorias de competência motora e EMT. A análise da fidedignidade foi investigada por meio da analise da Consistência Interna utilizando como índice o Alpha de Cronbach; e, da objetividade entre observadores utilizando-se 0 coeficiente de correlação intra-classe. O nível de significância adotado foi de $p \leq 0,05$ e nas análises de grupos idade $\mathrm{p}=0,01$ pela correção de Bonferroni. Coeficientes de correlação acima de 0,60 foram considerados fortes; entre 0,30 e 0,60 : moderados; e, abaixo de 0,3: pobres (VALLERAND, 1989). Para o Alpha de Cronbach, valores acima de 0,80 ; foram considerados ótimos; acima de 0,70 bons; e, entre 0,60 e 0,70, aceitáveis (DASSA, 1999).

\section{Resultados}

\section{Tradução}

$\mathrm{Na}$ versão final e unificada da LC-MABC-2, o comitê reunido consolidou todas as versões produzidas, 4 traduções do questionário original, e desenvolveu a versão final que foi utilizado no teste de campo (BEATON et. al., 2000). A semântica dos itens foi mantida. Mudanças foram necessárias quanto ao uso de palavras mais frequentes no cotidiano e correções de termos técnicos utilizados na tradução; tendo em consideração a compreensão do instrumento pelo público alvo (exemplos: pracinha, pega-pega).

\section{Validade de Conteúdo e de Face}

O painel de especialistas e o publico alvo (especificamente os professores de educação física e fisioterapeutas) envolvidos no processo de validação do instrumento questionaram a praticidade de observar e a adequação para a realidade escolar brasileira de dois itens da seção $B$ da lista de checagem original (B.3.1. Anda de bicicleta sem rodinhas e B.3.3. Mantém o equilíbrio na água entre outras crianças). Após pesquisas de atividades do contexto infantil, novos itens foram delineados pelos pesquisadores os quais buscaram aproximar da realidade das crianças brasileiras $\mathrm{e}$ das demandas motoras dos itens originais (B.3.1. Anda sobre latas, uma em cada pé preza por uma corda [pé de lata] e B.3.3. Mantém o equilíbrio saltitando com outras crianças sob uma corda que se movimenta de um lado para o outro [cobrinha, minhoquinha]).

A versão em português, e adaptada, do LCMABC-2 foi enviada novamente ao painel de especialistas e ao público alvo. Especialistas, professores, terapeutas e pais consideraram que todos os itens estavam apropriados para os propósitos de mensuração, inclusive os dois novos itens adaptados. Reportaram que as questões que compõem a LC-MABC-2 pertencem ao cotidiano das crianças; são práticas pertinentes, e representam um conjunto de tarefas que são coerentes com as demandadas do contexto infantil.

Quanto à validade de conteúdo (Tabela 2), para clareza, os valores de concordância foram elevados para o IVC e por associações significativas entre os escores do painel de especialistas. Sugestões de alterações quanto à linguagem e inserção de exemplos oferecidos pelo painel de especialistas foram incorporadas na versão final do instrumento. Para a pertinência, a concordância entre avaliadores foi acima de 90\% para a maioria das seções e itens do instrumento. Para a clareza e pertinência observou-se concordância ótima e não diferença significante entre os escores (valores de $p \geq$ $0,05)$.

\section{Validade de Construto: Convergente e Discriminante}

Quanto à Validade Convergente para o total da amostra foi encontrada uma correlação positiva, significativa e forte entre seção $A$ e seção $B$. Nos grupos de idades as correlações foram positivas, moderadas e significativas (exceção para 5, 8 e 12 anos) (Tabela 3). 
Tabela 2. Índice de Validade de Conteúdo (IVC) e resultados de testes de comparações.

\begin{tabular}{|c|c|c|c|c|c|c|c|c|c|c|c|c|}
\hline \multirow{3}{*}{ Seções } & \multicolumn{12}{|c|}{ CLAREZA } \\
\hline & \multicolumn{3}{|c|}{ Experts $1 \times 2$} & \multicolumn{3}{|c|}{ Experts $1 \times 3$} & \multicolumn{3}{|c|}{ Experts $2 \times 3$} & \multicolumn{3}{|c|}{ Experts $1 \times 2 \times 3$} \\
\hline & IVC \% & $Z$ & $p$ & IVC \% & Z & $P$ & IVC \% & $Z$ & $P$ & IVC \% & $\mathrm{F}$ & $P$ \\
\hline A & 80 & $-1,73$ & 0,08 & 93 & $-1,00$ & 0,32 & 87 & $-1,63$ & 0,10 & 80 & 5,60 & 0,06 \\
\hline B & 87 & $-1,41$ & 0,16 & 87 & $-1,41$ & 0,16 & 87 & 0,001 & 1,00 & 73 & 2,67 & 0,26 \\
\hline \multirow[t]{3}{*}{$\mathrm{C}$} & 85 & $-1,00$ & 0,32 & 85 & $-1,32$ & 0,18 & 77 & 0,001 & 0,101 & 77 & 2,00 & 0,37 \\
\hline & \multicolumn{12}{|c|}{ PERTINÊNCIA } \\
\hline & \multicolumn{3}{|c|}{ Expert $1 \times 2$} & \multicolumn{3}{|c|}{ Expert $1 \times 3$} & \multicolumn{3}{|c|}{ Expert $2 \times 3$} & \multicolumn{3}{|c|}{ Expert $1 \times 2 \times 3$} \\
\hline Seções & IVC \% & Z & $p$ & IVC \% & Z & $p$ & IVC \% & Z & $P$ & IVC \% & $\mathrm{F}$ & $P$ \\
\hline$A$ & 93 & $-1,00$ & 0,32 & 93 & $-1,00$ & 0,10 & 93 & $-1,00$ & 0,32 & 87 & 2,00 & 0,37 \\
\hline B & 87 & 0,001 & 1,00 & 93 & 0,001 & 0,10 & 93 & 0,001 & 1,00 & 87 & 0,001 & 1,00 \\
\hline C & 93 & $-1,00$ & 0,32 & 93 & $-1,00$ & 0,32 & 93 & 0,001 & 1,00 & 85 & 1,00 & 0,61 \\
\hline
\end{tabular}

Nota: Z: Teste de Wilcoxon; F: Teste de Friedman

Tabela 3. Seção A e B e EMT: Correlações e Comparações entre Seções e idade

\begin{tabular}{|c|c|c|c|c|c|c|c|c|}
\hline & \multicolumn{2}{|c|}{ Seção A } & \multicolumn{2}{|c|}{ Seção B } & \multicolumn{2}{|l|}{ EMT } & \multicolumn{2}{|c|}{ Seção A x Seção B } \\
\hline & $M(D P)$ & Md & $M(D P)$ & Md & $M(D P)$ & Md & $z(p)$ & $r(\mathrm{p})$ \\
\hline \multicolumn{9}{|c|}{ Total Amostra } \\
\hline & $2,70(4,77)$ & 0 & $4(5,33)$ & .0 & $6,71(9,38)$ & 2 & $-8,42(0,001)$ & $0,72(0,001)$ \\
\hline \multicolumn{9}{|c|}{ Grupos de Idade } \\
\hline 5 & $15,32(4,23)$ & 15 & $17,96(5,03)$ & 15 & $33,28(7)$ & 31 & $-2,03(0,04)$ & $0,14(0,24)$ \\
\hline 6 & $8,40(4,20)$ & 8 & $8,63(4,31)$ & 7 & $17,03(6,78)$ & 15 & $-0,41(0,68)$ & $0,27(0,02)$ \\
\hline 7 & $4,67(4,12)$ & 4 & $4,61(4,26)$ & 4 & $0,28(7,39)$ & 8 & $-0,81(0,42)$ & $0,55(0,001)$ \\
\hline 8 & $0,57(1,66)$ & 0 & $5,74(4,38)$ & 5 & $6,30(4,95)$ & 5 & $-7,1(0,001)$ & $0,17(0,07)$ \\
\hline 9 & $0,67(1,46)$ & 0 & $2,13(2,49)$ & 1 & $2,80(3,48)$ & 2 & $-5,78(0,001)$ & $0,51(0,001)$ \\
\hline 10 & $0,46(1,01)$ & 0 & $1,21(1,75)$ & 0 & $1,66(2,49)$ & 1 & $-4,03(0,001)$ & $0,59(0,001)$ \\
\hline 11 & $0,04(0,35)$ & 0 & $0,47(1,06)$ & 0 & $0,51(1,24)$ & 0 & $-3,77(0,001)$ & $0,39(0,001)$ \\
\hline 12 & $0,21(1,58)$ & 0 & $0,19(0,51)$ & 0 & $0,40(1,64)$ & 0 & $-1,89(0,06)$ & $-0,05(0,35)$ \\
\hline$K W(p)$ & $367(0,00$ & & $319(0,00$ & & $348(0,00$ & & -- & -- \\
\hline
\end{tabular}

$\mathrm{Na}$ analise da Validade Discriminante três procedimentos foram adotados. Primeiramente, seguindo o delineamento original (HENSERSON et al., 2007) verificou se a seção B de fato se diferencia na seção $A$ em termos de dificuldade motora, uma vez que as mesmas envolvem movimentação. Os resultados do teste de Wilcoxon evidenciaram diferenças significativas entre os escores da seção A e B para o total da amostra e para a maioria dos grupos de idades, com exceção nos grupos de 6, 7 e 12 anos (Tabela 4).

Tabela 4. Objetividade Inter-Avaliadores

\begin{tabular}{lccc}
\hline \multirow{2}{*}{ Avaliadores } & \multicolumn{3}{c}{ Objetividade Inter-Avaliadores (A) } \\
\cline { 2 - 4 } & Seção A ICC & Seção B ICC & Seção C ICC \\
\hline Profs. Ed. Física x Ed. Física & $0,90^{\prime} ; 0,89^{2} ; 0,91^{3}$ & $0,83^{\top} ; 0,81^{2} ; 0,82^{8}$ & $0,78^{\prime} ; 0,79^{2} ; 0,76^{3}$ \\
\hline Prof. Sala Aula x Pais & 0,82 & 0,84 & 0,47 \\
\hline Profs. Sala Aula x Ed. Física & 0,83 & 0,78 & 0,56 \\
\hline Prof. Ed. Física x Pais & 0,85 & 0,85 & 0,60 \\
\hline
\end{tabular}

Nota: ${ }^{1} \mathrm{~A} 1 \times \mathrm{A} 2 ;{ }^{2} \mathrm{~A} 1 \times \mathrm{A} 3 ;{ }^{3} \mathrm{~A} 2 \times \mathrm{A} 3$.

O segundo procedimento envolveu investigar se crianças com RAM e DMG se diferenciaram em desempenho quando comparadas aos seus pares $\mathrm{CM} ; 86,5 \%$ das crianças foram categorizadas como CM; 8,3\% em RAM; e, 5,3\% com DMG. Diferenças significativas foram observadas, $(H(2)=62,17, P=0,0001)$, entre os EMT das crianças que demonstraram CM, RAM e DMG. Mann Whitney post hoc testes evidenciaram que crianças CM (M Rank $=242$ ) demonstraram desempenho significativamente 
superior, $z=-5.22, p=0,0001$, as crianças do grupo RAM (M Rank = 359). As crianças com DMG (M Rank =403) demonstraram desempenhos inferiores aos seus pares do grupo CM, $z=-6,28, p=0,0001$ e do grupo RAM , $z=-$ 2.42, $p=0,02$.

O terceiro procedimento envolveu analisar a associação entre as categorias de competência motora e o EMT utilizando-se do Eta e $\mathrm{Eta}^{2}$ (associação entre variáveis qualitativas e quantitativas). Os resultados evidenciam uma associação de variação moderada $\eta=0,33$ e $\eta^{2}$ $=0,57$.

\section{Fidedignidade: Consistência interna e objetividade}

Quanto à análise de Consistência Interna o coeficiente de confiabilidade Alpha de Cronbach para o os itens da LC-MABC-2 demonstrou ótima confiabilidade $(\alpha=0,94)$; e, avaliados os itens em seção, os valores se mantiveram altos para a Seção $A(\alpha=0,95)$ e Seção $B(\alpha=0,94)$, entretanto, para a Seção $C$, o resultado foi considerado somente aceitável $(\alpha=0,60)$. Para a soma dos escores, a confiabilidade foi confirmada nas 3 seções $(\alpha=0,79)$.

A objetividade entre avaliadores foi investigada com os professores de Educação Física responsáveis por avaliar as crianças no presente estudo. E em 20 crianças a objetividade entre professores de sala de aula, pais e um professor de educação física foi também investigada (Tabela 4). Os coeficientes de correlação intraclasse, indicaram forte concordância entre avaliadores.

\section{Tendência desenvolvimentista}

Médias e medianas decrescem com a idade. Comparações nas idades em cada seção especifica da LC-MABC-2 evidenciou que diferenças significativas foram encontradas nos grupos de idade para as Seções A, B e EMT (Tabela 3). Testes de continuidade de Mann Whitney evidenciaram diferenças significativas para a maioria dos grupos de idade (valores de $p$ $\leq 0,01$ adotando correção de Bonferroni). Exceção foram observadas nas comparações na Seção A entre crianças de 8 e 10 anos $(p=0,45)$ e 9 e 10 anos ( $p=0,38)$; e, na Seção $B$ entre crianças de 7 e 8 anos $(p=0,08)$. Para crianças de 11 e 12 anos, não foram observadas diferenças significativas para EMT $(p=0,38)$, seção $A$ $(p=0,84)$ e seção $B(p=0,23)$.

\section{Discussão}

As propriedades psicrométricas do LC-MABC2 foram consideradas com adequada confiabilidade (BEATON, et al., 2000; CRONBACH, 1996; FACHEL; CAMEY, 2003; PASQUALI, 2001; VALLERAND, 1989). A abordagem transcultural com tradução reversa e independente resultou em uma versão adaptada unificada em língua português. A comparação realizada entre as quatro versões traduzidas, a discussão e análise em comitê possibilitou a redução de vieses e da ocorrência de diferentes significados, inerentes ao processo com um só tradutor (BEATON et al., 2000; FACHEL; CAMEY, 2003; HERNANDEZ-NIETO, 2002).

Quanto a Validade de Conteúdo, no que se refere à concordância das avaliações do painel de experts quanto ao IVC para Clareza e Pertinência, observa-se uma concordância forte entre os juízes e semelhanças nas respostas, confirmada por associações significativas (BEATON et al., 2000; CRONBACH, 1996; VALLERAND, 1989). O LC-MABC-2 apresentou índices ótimos de validade de conteúdo, apresentando critérios claros e pertinentes, adequada representação dos itens em relação aos conceitos e a relevância teórica (CRONBACH, 1996; DASSA, 1999; PASQUALI, 2001).

Quanto à Validade de Face, os profissionais foram unânimes em indicar o conteúdo da LCMABC-2 como adequada para avaliar as habilidades motoras de crianças. Destaca-se que é fundamental que um instrumento contenha itens práticos, claros e adequados ao contexto de desenvolvimento, ainda mais considerando-se a perspectiva dos adultos (pais, terapeutas e professores) que vão avaliar essas crianças, resultado observado no presente estudo (PASQUALI, 2001; VALLERAND, 1989).

Para a Validade de Construto, verificou-se, que quanto a Validade Convergente os escores da Seção $A$ se encontram fortemente associados aos escores da seção $B$ para o total da amostra, e moderadamente nos vários grupos de idades. $O$ cálculo de um escore total só é justificado quando os itens, embora mensurando, diferentes habilidades apresentem relação entre si (CRONBACH, 1996; DASSA, 1999; FACHEL; CAMEY, 2003; HERNANDEZ-NIETO, 2002). As correlações observadas entre escores na Seção A e Seção B, para toda a amostra bem como para a maioria dos grupos de idades reforçam 0 
entendimento teórico da relação entre seções e, portanto este resultado suporta o uso do EMT; resultado semelhante ao reportado na validação original da LC-MABC-2 (HENDERSON et al., 2007). A associação positiva e moderada entre as duas medidas de um mesmo constructo confirmam a validade convergente (FACHEL; CAMEY, 2003). Entretanto, se observa que a magnitude das correlações obtidas no presente estudo são mais fracas que as observadas na validação do teste com crianças inglesas (HENDERSON et al., 2007). Ainda mais, para crianças com 12 anos, o EMT deve ser utilizado com cuidado, ou ainda não utilizado, uma vez que não foram observadas diferenças nem correlações significativas entre escores da seção $A$ e seção $B$.

Quanto a Validade Discriminante, os resultados evidenciaram que a Seção $B$ avalia níveis mais elevados de imparidade motora, resultados reportados em estudos prévios (HENDERSON et al., 2007). Destaca-se que atividades realizadas em um ambiente dinâmico, como as tarefas em movimentação da Seção B foram delineados para requererem níveis mais complexos de habilidade quando comparadas as tarefas propostas na seção A, resultado confirmado no presente estudo.

Os resultados indicaram que o LC-MABC-2 é válido para discriminar desempenho de $\mathrm{CM}$, RAM e DMG. O EMT foi significativamente diferente nos grupos investigados. Ainda mais que houve associação moderada entre EMT e categorização; sugerindo que escores elevados no EMT estão associados com dificuldades motoras graves enquanto que escores mais baixos com desempenhos mais competentes. Portanto, considerando o critério de categorização a validade discriminante se confirma, diferenciando os grupos (FACHEL; CAMEY, 2003) similar a estudos prévios (HENDERSON et al., 2007).

Com relação à objetividade os resultados demonstram concordância forte entre os avaliadores (ICC entre 0,78 e 0,91 ) para as seções A e B. Os altos índices de confiabilidade, principalmente na seção $A$, evidenciam a concordância adequada (DASSA, 1999; HERNANDEZ-NIETO, 2002; VALLERAND, 1989). Na Seção C, a objetividade entre avaliadores com uma mesma formação acadêmica foi alta (entre professores de educação física); entretanto, a objetividade entre pais e professores e entre professores de sala de aula e educação física pode ser considerada frágil e apenas aceitável (DASSA,1999; HERNANDEZ-NIETO, 2002) evidenciando que as razões para os atrasos motores foram identificadas de maneira diferenciada. Sugere-se que este público alvo (professores de sala de aula e pais) poderiam realizar estas observações acompanhados de um profissional treinado, e ou ainda após treinamento por um profissional da área da saúde.

\section{Distribuição por idades e tendências desenvolvimentais}

O EMT decresce significativamente com a idade (Tabela 3). Ao se observar as médias e principalmente as medianas verifica-se que 0 efeito chão, reportado pelos autores do teste (HENDERSON et al., 2007) ocorre a partir de 8 anos para a Seção $A$; para a seção $B$ aos 10 anos; e, para o EMT a partir dos 11 anos. Entre 11 e 12 anos não são observadas diferenças significativas de desempenho, evidenciando um platô mais tardio que observado em crianças inglesas (HENDERSON et al., 2007). Este efeito é esperado uma vez que a LC-MABC-2 foi desenvolvida para diferenciar crianças com dificuldades motoras, e contém itens que crianças de desenvolvimento típico podem realizar com relativa facilidade nos primeiros anos escolares (HENDERSON et al., 2007). A LC-MABC-2 foi desenvolvida com o objetivo de avaliar crianças de 5 a 12 anos, e se fundamenta no entendimento de que a competência motora de crianças aumenta consideravelmente neste período (HENDERSON et al., 2007). Observa-se que os resultados do presente estudo confirmam esta trajetória desenvolvimentista.

\section{Consistência Interna}

A LC-MABC-2 possui elevada consistência interna; os valores do Alpha de Cronbach (0,72 a $0,89)$ refletem um perfil de alta homogeneidade entre as variáveis. Os valores acima de 0,70, mais difíceis de serem obtidos em amostras maiores refletem 0 quanto os itens são homogêneos e que constituem a representação de um mesmo traço; medindo o mesmo construto (BEATON, et. al., 2000; CRONBACH, 1996; DASSA, 1999).

Concluindo, os resultados evidenciaram uma tradução eficiente para evitar vieses da compreensão da língua inglesa. Os resultados se mostraram fidedignos, consistentes e com poder discriminante adequado. Crianças avaliadas como 
demonstrando dificuldades motoras na LC-MABC2 devem ser encaminhadas para avaliações mais especificas com testes normatizados. O presente estudo reporta adequação das capacidades psicrométricas da LC-MABC-2 e reforça a importância de capacitar profissionais no uso de instrumentos de triagem validados para as crianças brasileiras.

\section{Referências}

AMERICAN PSYCHIATRIC ASSOCIATION. DSM-IV-TR Diagnostic and Statistical Manual of Mental Disorders. Washington, DC: American Psychiatric Association, 2000.

BEATON, D. E.; BOMBARDIER, C.; GUILLEMIN, F.; FERRAZ, M. B. Guidelines for process of cross-cultural adaptation of self-report measures. Spine, Lebanon, v. 25, n. 24, p. 3186-3191, 2000.

BARNETT, A. L.; SUGDEN, D. A.; HENDERSON, S. E. Review of the Movement ABC Checklist Second Edition. In: $8^{\text {th }}$ Motor Control and Human Skill Conference, 2007, Fremantle.Anais do $8^{\text {th }}$ Motor Control and Human Skill Conference, p.250, 2007.

CAIRNEY, J.; HAY, J. A.; VELDHUIZEN, S.; MISSIUNA, C.; FAUGHT, B. E. Developmental coordination disorder, sex, and activity deficit over time: a longitudinal analysis of participation trajectories in children with and without coordination difficulties. Developmental Medicine \& Child Neurology, Oxford, v.52, n. 3, p.67-72, 2010. http://dx.doi.org/10.1111/j.14698749.2009.03520.x

COUSINS, M.; SMYTH, M. M. Developmental coordination impairments in adulthood. Human Movement Science, Amsterdam, v. 22, p. 433459, 2003.

CRONBACH, L. J. Fundamentos da testagem psicológica. Porto Alegre: Artes Médicas; 1996.

CUMMINS, A.; PIEK, J. P.; DYCK, M. J. Motor coordination, empathy, and social behaviour in school-aged children. Developmental Medicine \& Child Neurology, Oxford, v. 47, n. 7, p. 437442, 2005. Disponível em: http://www.ocogym.com/pdf/pdf 15.pdf Acesso em 25 set. 2011.

\section{DASSA, C. Analyze multidimensionnelle} exploratoire et confirmative. Montreal, Univesité de Montreal, 1999.

DIDO, G. et. al. Is Questionnaire-Based Screening Part of the Solution to Waiting Lists for Children with Developmental Coordination Disorder? The British Journal of Occupational Therapy, London, v. 68, n. 1, p. 2-10, 2005. Disponível em: http://www.ingentaconnect.com/content/cot/bjot/20 05/00000068/00000001/art00002 Acesso em 11 set. 2011.

FACHEL, J. M. G.; CAMEY, S. Avaliação Psicométrica: A qualidade das medidas e 0 entendimento dos dados. In: J. A. Cunha e colaboradores (Orgs.), Psicodiagnóstico. Porto Alegre: Artmed; 2003.

FERREIRA, L. F.; SOUZA, C. J. F.; ARAUJO, U. $O$. Estudo correlacional entre a seção cinco e demais seções da lista de checagem do teste MABC. Motriz Revista de Educação Física. Rio Claro, v. 14, n. 1, p.21-29, 2008a. Disponível em: http://www.periodicos.rc.biblioteca.unesp.br/index. php/motriz/article/view/1357/1189. Acesso em 12 out. 2011.

FERREIRA, L.F.; SOUZA, C. J.

F.; FREUDENHEIM, A. M. A efetividade da lista de checagem do teste $A B C$ do movimento.

Revista Portuguesa Ciências do Desporto.

Porto, v. 8, n.3, p. 347-354, 2008b. Disponível em: http://www.scielo.gpeari.mctes.pt/pdf/rpcd/v8n3/v8 n3a05.pdf Acesso em 15 out. 2011.

GEUZE, R. H. Postural Control in Children with Developmental Coordination Disorder. Neural Plasticity. New York, v.12, p.183-196, 2005. Disponível em: http://www.hindawi.com/journals/np/2005/831549/ abs/Acesso em 15 set. 2011.

GIBBS, J.; APPLETON, J.; APPLETON, R. Dyspraxia or developmental coordination disorder? Unravelling the enigma. Archives of Disease in Childhood. London, v. 92, p.534-539, 2007. http://dx.doi.org/10.1136/adc.2005.088054

HENDERSON, S. E.; SUGDEN, D. A.; BARNETT, A. L. Movement assessment battery for children-2 second edition [Movement ABC-2]. London, UK: The Psychological Corporation, 2007.

HERNANDEZ-NIETO, R. Contributions to statistical analysis. Mérida: Los Andes University Press, 2002.

MAHONEY, G.; ROBINSON, C.; PERALES, F. Early Motor Intervention - The Need for New Treatment Paradigms. Infants and Young Children, Philadelphia, v.17, n. 4, p. 291-300, 2004. Disponível em: http://depts.washington.edu/isei/iyc/mahoney 17 4.pdf Acesso em: 27 abr. 2011.

MISSIUNA, C.; MOLL, S.; KING, S.; KING, G.; LAW, M. A trajectory of troubles: Parents' impressions of the impact of developmental coordination disorder. Physical \& Occupational Therapy in Pediatrics, Philadelphia, v. 27, n. 1, p. 
81-101, 2007. Disponível em:

http://www.ncbi.nlm.nih.gov/pubmed/17298942

Acesso em: 12 set. 2011.

PASQUALI, L. Parâmetros psicométricos dos testes psicológicos. São Paulo: Casa do Psicólogo Livraria e Editora; 2001.

POULSEN, A. A.; ZIVIANI J. M.; CUSKELLY M.; SMITH R. Boys with developmental coordination disorder: Loneliness and team sport participation. American Journal of Occupational Therapy, Bethesda, v. 61, n. 4, p. 463-474, 2007.

Disponível em: http://www.ncbi.nlm.nih.gov/pubmed/17685178 Acesso em 19 abr. 2011.

PRADO, M. S.S.; MAGALHÃES, L. C.; WILSON, B. N. Adaptação transcultural do Questionário de Transtorno do Desenvolvimento da Coordenação para crianças brasileiras. Revista Brasileira de Fisioterapia, São Carlos, v.13, n. 3, p. 236-43, 2009. Disponível em: http://www.physiokinder.de/news/DCDQ\%20Artikel\%20Monja.pdf Acesso em: 17 abr. 2011.

SCHOEMAKER, M. M. Manual of the motor observation questionnaire for teachers. Groningen: Internal Publication, Center for Human Movement Sciences, Dutch, 2003.

SCHOEMAKER, M. M.; SMITS-ENGELSMAN, B. C. M.; JONGMANS, M. J. Psychometric properties of the Movement Assessment Battery for Children-Checklist as a screening instrument for children with a developmental co-ordination disorder. British Journal of Educational Psychology, Malden, v. 73, n. 3, p. 425-441, 2003. Disponível em http://www.ncbi.nlm.nih.gov/pubmed/14672152 Acesso em: 18 mar. 2011.

SCHOTT, N.; ALOF, V.; HULTSCH, D.; MEERMANN, D. Physical fitness in children with developmental coordination disorder. Research Quarterly for Exercise \& Sport, Reston, v. 78, n.5, p. 438-450, 2007. Disponível em: http://www.ncbi.nlm.nih.gov/pubmed/18274216 Acesso em: 20 jan. 2011.

SIAPERAS, P.; HOLLAND, T.; T. RING, H.

Discriminative validity of the Movement $A B C$ test and checklist for use with children with Asperger Syndrome. In S. E. HENDERSON, D. SUGDEN; A. BARNETT, Movement Assessment Battery for Children - second edition London: Pearson Assessment, 2007. p. 157-159.

SILVA, J. A. O; DANTAS, L. E.; CATTUZZO, M. T.; WALTER, C.; MOREIRA, C. R .P.; SOUZA, C. J. F. Teste MABC: aplicabilidade da lista de checagem na região sudeste do Brasil. Revista Portuguesa Ciências do Desporto, Porto, v. 6, n.3, p. 356-361, 2006. Disponível em:

http://www.scielo.gpeari.mctes.pt/pdf/rpcd/v6n3/v6 n3a12.pdf Acesso 18 abr. 2011.

SUMMERS, J. M.; LARKIN, D.; DEWEY, D. Activities of daily living in children with Developmental Coordination Disorder: Dressing, Personal Hygiene and, Eating Skills. Human Movement Science, Amsterdam, v. 27, p. 215229, 2008. Disponível em:

http://www.ncbi.nlm.nih.gov/pubmed/18348898

Acesso em: 12 abr. 2011

VALENTINI, N. C.; BARBOSA, M. L. L.; CINI, G. V.; PICK, R. K. SPESSATO, B. C.; BALBINOTTI, M. A. A. Test of Gross Motor Development: expert validity, confirmatory validity and internal consistence. Revista Brasileira de Cineantropometria e Desempenho Humano, Florianópolis, v. 10, n. 4, p. 399-404, 2008. Disponível em:

http://www.periodicos.ufsc.br/index.php/rbcdh/artic le/view/1980-0037.2008v10n4p399 Acesso em 12 abr. 2011.

VALLERAND, R. J. Vers une méthodologie de validation trans-culturelle de questionnaires psychologiques: implications pour la recherche en langue française. Canadian Psychology, v. 30, n. 4, p. 662-680, 1989.

http://dx.doi.org/10.1037/h0079856

VISSER, J. Developmental coordination disorder: a review of research on subtypes and comorbidities. Human Movement Science, Amsterdam, v. 22, n. 4-5, p. 479-493, 2003. http://dx.doi.org/10.1016/j.humov.2003.09.005

\section{Apoio Financeiro: CAPES e CNPq}

\section{Endereço:}

Nadia C. Valentini

Rua Felizardo Furtado, 750 Jd. Botânico

Porto Alegre RS Brasil

90690-200

Telefone: (51) 3308.5856

e-mail: nadiacv@esef.ufrgs.br

Recebido em: 4 de novembro de 2011.

Aceito em: 8 de maio de 2013.

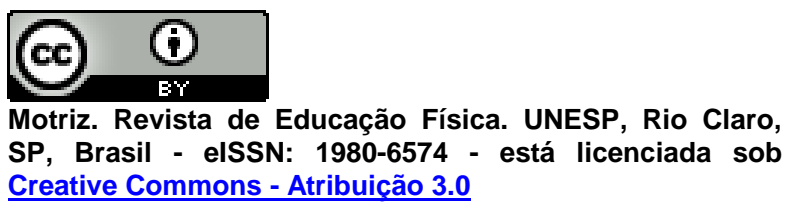

